\title{
NONLINEAR DAMPING OF INJECTION OSCILLATIONS
}

\author{
V. M. Zhabitsky \\ Joint Institute for Nuclear Research, 141980 Dubna, Moscow region, Russia
}

\section{Abstract}

The theory of a transverse feedback system with a nonlinear regime of injection oscillations damping in circular accelerators and colliders is described. For this feedback loop the dependence between the kick value in the deflector and the beam deviation at the pick-up location is a nonlinear function. The beam dynamic nonlinear equation of the transverse coherent motion for deviation from the closed orbit has been solved using the approximation procedure based on the Krylov-Bogoliubov method for nonlinear differential equations. Approximate expressions for damping time and beam oscillation amplitude are analysed for different nonlinear transfer functions.

\section{INTRODUCTION}

Transverse feedback systems (TFS) are used in synchrotrons to damp the coherent transverse beam oscillations. In these systems (see Fig.1) the kicker (DK) corrects the beam angle according to the beam deviation from the closed orbit in the pick-up (PU) location at every turn. TFS have been widely used to suppress resistive wall instability and to provide a beam oscillation amplitude decrease after injection.

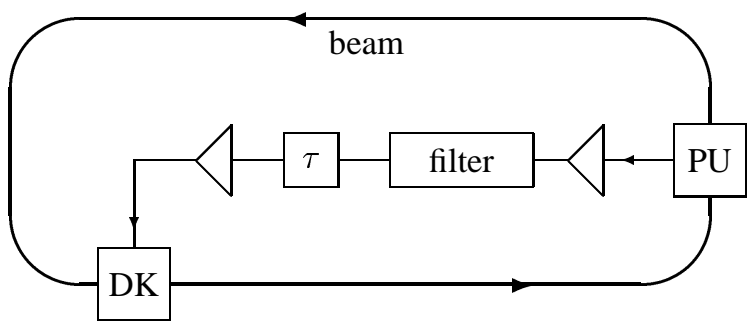

Figure 1: Layout of a transverse feedback system

Most TFS use electronics for signal processing in the feedback loop in order to obtain different dependences $f(x)$ between the beam deviation $x\left[n, s_{K}\right]$ in PU and the kick $\Delta x^{\prime}\left[n, s_{K}\right]$ in DK at the $n$-th turn:

$$
\sqrt{\beta_{P} \beta_{K}} \Delta x^{\prime}\left[n, s_{K}\right]=g f\left(x\left[n, s_{P}\right]\right) .
$$

Here $\beta_{P}$ and $\beta_{K}$ are the transverse betatron amplitude functions in the PU and DK locations; $g$ is the gain of the feedback loop. Usually power amplifiers with a linear characteristic are employed. Hence, a transfer function $f(x)$ of this feedback loop is a linear one (see Fig.2, dashed line).
However, many TFS use digital electronics in the feedback loop (for example, filters and delays). Their transfer functions have a quasi-linear step character. Sometimes the regime with limitation of a power for amplifiers is employed for large amplitudes of beam oscillations. On the SPS at CERN, the "bang-bang" regime was realized to increase the efficiency of TFS [1]. Its transfer function consists of a linear part for small amplitude oscillations and a high fixed level gain for large oscillations (see Fig.2, solid line). Thus, the transfer functions $f(x)$ of these TFS are nonlinear.

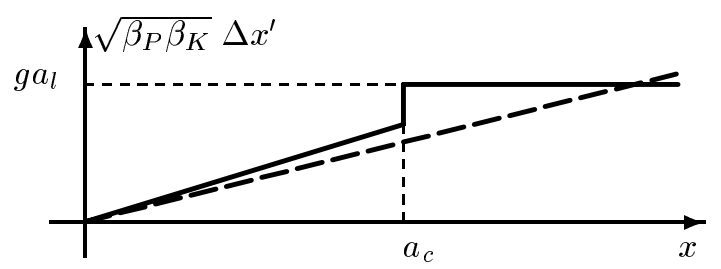

Figure 2: Dependence of the kick $\Delta x^{\prime}$ on the beam deviation $x$ for the linear (dashed line) and "bang-bang" (solid line) transfer functions.

The so-called "logical regime" was described in [2]. Its transfer function is a step function with two jumps. This nonlinear regime was tested on the SPS at CERN [3]. The fast damping of initial oscillations was observed.

These experiments initiated some theoretical studies. A numerical simulation was used in [2] to estimate the damping time. It has been found that the amplitude of oscillations decreases linearly in time for some regimes.

The analitical appoach for nonlinear damping was developed in [4]. It is shown that the transverse coherent motion for the particle deviation from the closed orbit, when instability is neglected, is fully described by the difference equation of the second order:

$$
\begin{aligned}
x[n & \left.+2, s_{P}\right]-2 x\left[n+1, s_{P}\right] \cos \mu+x\left[n, s_{P}\right] \\
= & \sqrt{\beta_{P} \beta_{K}} \Delta x^{\prime}\left[n+1, s_{K}\right] \sin (\mu-\eta) \\
& +\sqrt{\beta_{P} \beta_{K}} \Delta x^{\prime}\left[n, s_{K}\right] \sin \eta
\end{aligned}
$$

where $\mu=2 \pi Q$ is a betatron phase advance per revolution in the transverse plane, $Q$ is the number of unperturbed betatron oscillations per revolution, and $\eta$ is the betatron phase advance from PU to DK.

To solve equation (2), the approximation procedure based on the Krylov-Bogoliubov method [5] for nonlinear 
differential equations was used in $[4,6]$. These approximation approaches for nonlinear damping are generalized below. Damping time and asymptotic behaviour of the beam oscillation amplitude are analysed for different parameters of the nonlinear transfer function. All results are obtained for feedback description when instability is neglected.

\section{THEORY}

\subsection{Basic Equation}

In order to simplify final expressions, it will be supposed further that the phase advance $\eta$ from the PU to the DK is equal to an odd number of $\pi / 2$ radians. So, $|\sin \eta|=1$ and the best damping is realised for TFS with the linear transfer function. Substituting for $\Delta x^{\prime}$ from (1) into (2) in this case yields

$$
\begin{aligned}
& x\left[n+2, s_{P}\right]-2 x\left[n+1, s_{P}\right] \cos \mu+x\left[n, s_{P}\right] \\
& =g f\left(x\left[n, s_{P}\right]\right)-g f\left(x\left[n+1, s_{P}\right]\right) \cos \mu .
\end{aligned}
$$

Equation (3) is a basic equation for studying beam dynamics with a nonlinear transfer function for a feedback loop. This equation is good for numerical calculations and convenient for analytical work.

\subsection{Solution (First Approximation)}

The gain $g$ in (3) for feedback realized is a small value. Normally, $g \approx 0.01$ for instability damper systems and $g \approx 0.1$ for damping of injection errors. Since $g$ is small, equation (3) is weakly nonlinear, and a number of perturbation methods is available to determine an approximate solution of this equation. It was demonstrated in $[4,6]$ that the Krylov-Bogoliubov method [5] can be used for solving the weakly nonlinear equation (3). This approach is used and generalized below.

When $g=0$, the solution of (3) can be written as

$$
\begin{aligned}
x\left[n, s_{P}\right] & =a \cos (\mu n+\phi)=a \cos \psi_{n}, \\
\psi_{n} & =\mu n+\phi,
\end{aligned}
$$

where $a$ and $\phi$ are constants. When $g \neq 0$, the solution of (3) can still be expressed in form (4), provided that $a$ and $\phi$ are considered to be functions of $n$ rather than constants. In accordance with the Krylov-Bogoliubov method, the solution of (3) can be written as a series of the form

$$
x\left[n, s_{P}\right]=a_{n} \cos \psi_{n}+\sum_{m=1}^{\infty} g^{m} \xi_{m}\left(a_{n}, \psi_{n}\right),
$$

where $\xi_{i}$ is unknown functions of full amplitude $a_{n}$ and periodical functions of $\psi_{n}$. Functions $\xi_{i}$ are small corrections of the main harmonic $a_{n} \cos \psi_{n}$. The order of these corrections is given by small parameter $g$. The amplitude and phase are the functions of $a_{n}$. Hence, for their derivatives we can write:

$$
\begin{aligned}
& \frac{d a_{n}}{d n}=g A_{1}\left(a_{n}\right)+g^{2} A_{2}\left(a_{n}\right)+\ldots \\
& \frac{d \psi_{n}}{d n}=\mu+g \Phi_{1}\left(a_{n}\right)+g^{2} \Phi_{2}\left(a_{n}\right)+\ldots .
\end{aligned}
$$

Functions $\xi_{m}$ as the periodical functions of $\psi_{n}$ can be expanded into the Fourier series:

$$
\begin{aligned}
& \xi_{m}\left(a_{n}, \psi_{n}\right)=\nu_{m 0}\left(a_{n}\right) \\
& \quad+\sum_{k=2}^{\infty}\left[\nu_{m k}\left(a_{n}\right) \cos k \psi_{n}+\gamma_{m k}\left(a_{n}\right) \sin k \psi_{n}\right],
\end{aligned}
$$

where $\nu_{m 1}=\gamma_{m 1}=0$, because amplitude $a_{n}$ is the full amplitude of the main (first) harmonic of oscillations.

For the left-hand side of (3) we expand all values into the Taylor series taking into account (6) and (7). The first approximation of these expansions is:

$$
\begin{aligned}
\text { l.h.s. } \simeq g A_{1}\left(a_{n}\right)\left[\cos \left(\psi_{n}+2 \mu\right)-\cos \psi_{n}\right] \\
+g \Phi_{1}\left(a_{n}\right) a_{n}\left[\sin \psi_{n}-\sin \left(\psi_{n}+2 \mu\right)\right] \\
+g \xi_{1}\left(a_{n}, \psi_{n+2}\right)-2 g \cos \mu \xi_{1}\left(a_{n}, \psi_{n+1}\right) \\
\quad+g \xi_{1}\left(a_{n}, \psi_{n}\right) .
\end{aligned}
$$

For the first level of approximation, the right-hand side of (3) is determined by a zero level of approximation. Substituting for $x$ from (5) into (3) yields

$$
\begin{array}{rl}
r . h . s .=g & f\left(a_{n} \cos \psi_{n}\right) \\
& -g f\left(a_{n} \cos \left(\psi_{n}+\mu\right)\right) \cos \mu .
\end{array}
$$

Equating coefficients of Fourier series in (8) and in (9) yields for main harmonic:

$$
\begin{aligned}
& \frac{d a_{n}}{d n}=-\frac{g}{2 \pi} \int_{0}^{2 \pi} f\left(a_{n} \cos \psi_{n}\right) \cos \psi_{n} d \psi_{n} \\
& \frac{d \psi_{n}}{d n}=\mu+\frac{g}{2 \pi a_{n}} \int_{0}^{2 \pi} f\left(a_{n} \cos \psi_{n}\right) \sin \psi_{n} d \psi_{n} .
\end{aligned}
$$

Equation (10) yields the amplitude damping rate per turn. The phase dependence on $n$ for the beam transverse oscillations is determined by equation (11).

It is clear from (8) and (9) that the third and higher harmonics of oscillations can be excited. It depends on the transfer function. This typical effect for nonlinear systems is not discussed in this paper because of pages limitation.

\section{RESULTS}

\subsection{Linear Transfer Function}

For TFS with a linear transfer function we have $f(x)=$ $x$, where $x=a_{n} \cos \psi_{n}$ at zero level of approximation. Taking into account (5), (10) and (11), we obtain the following solution:

$$
\begin{aligned}
x\left[n, s_{P}\right] & \simeq a_{0} \exp \left(-\frac{g}{2} n\right) \cos \psi_{n} \\
\psi_{n} & =\mu n+\phi_{0} .
\end{aligned}
$$

where $a_{0}$ and $\phi_{0}$ are constants depending on initial conditions. This solution coincides with the well known result (see, for example, [7]). 


\subsection{Transfer Function with Linear and Cubic Terms}

Performing the integration in (10) for the transfer function with linear and cubic terms

$$
g f(x)=g x+g g_{3} x^{3},
$$

we obtain:

$$
a_{n}=\frac{a_{0} \exp (-g n / 2)}{\sqrt{1-\left(3 g_{3} a_{0}^{2} / 4\right)(\exp (-g n)-1)}} .
$$

Performing the integration in (11), we obtain the expression for the phase of oscillations that coincides with formula (13). Thus, at the first level of approximation, the frequency is not affected by the damping, while the amplitude decreases in accordance with dependence (15). Formula (15) coincides with the well known result for the amplitude solution of Rayleigh's equation

$$
\ddot{x}+\omega_{0}^{2} x=\epsilon\left(\dot{x}-\lambda \dot{x}^{3}\right) .
$$

Other results concerning damping parameters for TFS with this transfer function are discussed in [4].

\subsection{Coulomb Damping}

In this case the transfer function is

$$
g f(x)=\left\{\begin{array}{rll}
g a_{l} & \text { when } & x_{n}>0 \\
-g a_{l} & \text { when } & x_{n}<0
\end{array}\right.
$$

Performing the integration in (10) and (11), we obtain formula (13) for the phase of oscillation and the following dependence on $n$ for the amplitude:

$$
a_{n}=a_{0}-\frac{2}{\pi} g a_{l} n .
$$

Thus, to this level of approximation, the amplitude decreases linearly with time. Formula (17) for amplitude dependence coincides with the well known result for amplitude solution of Coulomb damping nonlinear differential equation

$$
\ddot{x}+\omega_{0}^{2} x=\left\{\begin{array}{rll}
-g \lambda & \text { when } \quad x>0 ; \\
g \lambda & \text { when } \quad x<0,
\end{array}\right.
$$

where $\lambda=$ const.

\section{4 “Bang-Bang” Damping}

For TFS with a "bang-bang" transfer function we have (see Fig.2):

$$
g f(x)=\left\{\begin{array}{rll}
g x_{n} & \text { when }-a_{c} \leq x_{n} \leq a_{c} \\
g a_{l} & \text { when } x_{n}>a_{c} \\
-g a_{l} & \text { when } & x_{n}<-a_{c}
\end{array}\right.
$$

Therefore, from (10) we have for $a_{n}<a_{c}$

$$
\frac{d a_{n}}{d n} \simeq-\frac{g}{2} a_{n}
$$

and for $a_{n}>a_{c}$

$$
\begin{aligned}
\frac{d a_{n}}{d n} \simeq-\frac{g}{2 \pi}( & \left(4 a_{l}-2 a_{c}\right) \sqrt{1-\left(\frac{a_{c}}{a_{n}}\right)^{2}} \\
& \left.+\left(\pi-2 \arccos \left(\frac{a_{c}}{a_{n}}\right)\right) a_{n}\right)
\end{aligned}
$$

For the phase of oscillations we get formula (13). Hence, to the first level of approximation, the frequency is not affected by the damping, while the amplitude decreases in accordance with dependence (20) or (19).

For $a_{n} \gg a_{c}$ we have from (20)

$$
a_{n} \simeq a_{0}-\frac{2}{\pi} g a_{l} n
$$

Thus, to this level of approximation, large initial amplitudes decrease linearly with time. This formula (21) for amplitude dependence coincides with the result for amplitude solution of Coulomb damping (17). The linear amplitude decreasing with time was also obtained in [2] where a numerical simulation was used to estimate the damping time. Other results for the "bang-bang" regime are shown in [6].

\section{CONCLUSION}

The approaches demonstrated in $[4,6]$ have been developed and generalized in this paper for studying TFS with various nonlinear transfer functions. It gives analytical approximate solutions to calculate the damping time and other parameters of the particle motion.

\section{REFERENCES}

[1] X. Altuna et al. CERN Note SPS/ABM/RB/nb1, Geneva, 1987.

[2] I. N. Ivanov and V. A. Melnikov. Nonlinear damping of coherent oscillations of a beam in hadron cyclic accelerators and colliders. Nuclear Instruments and Methods in Physics Research, A 391(1):52-55, 1997.

[3] W. Höfle, I. N. Ivanov, R. Louwerse, and V. A. Melnikov. Suggestion to increase the transverse damper efficiency. Particle Accelerators, 58(1-4):281-286, 1997.

[4] V. M. Zhabitsky. Theory of a transverse feedback system with a nonlinear transfer function. In EPAC98. Proceedings of the Sixth European Particle Accelerator Conference, Stockholm, 22-26 June 1998, pages 1380-1382. IOP Publishing, Bristol and Philadelphia, 1998.

[5] N. M. Krylov and N. N. Bogoliubov. Introduction to Nonlinear Mechanics. Princeton University, 1947.

[6] V. M. Zhabitsky. Damping parameters of a transverse feedback system with a "bang-bang" transfer function. In HEACC'98. XVII International Conference on High Energy Accelerators. Dubna, 7-12 September 1998. JINR, E9-99-26, pages 391-393.

[7] V. M. Zhabitsky. Transverse feedback system with a digital filter. Nuclear Instruments and Methods in Physics Research, A 391(1):96-99, 1997. 